\title{
Elargissement du réseau de nos sociétés fiduciaires partenaires dans le canton du Jura
}

FMH Services présente un nouveau partenaire de confiance en matière fiduciaire dans le canton du Jura. Finest Gestion Sàrl a été créée en juin 2016 par Mario Fedi et Yann Burgener, directeurs associés. Tous deux sont en possession d'un brevet fédéral de spécialiste en finance et comptabilité et forts d'une expérience d'une vingtaine d'années chacun dans leurs domaines de prédilection (fiduciaire, fiscalité, gestion de PME, reporting financier, ressources humaines).

Notre société accorde une importance particulière au service personnalisé et à l'accompagnement de chaque client dans un climat de confiance réciproque. C'est en effet ainsi que nous nous sentons le plus à même de vous conseiller pour tout ce qui touche à vos questions financières. De même, afin de rendre la collaboration avec nos clients agréable, nous faisons preuve de flexibilité et de disponibilité.
Partenaires fiables dans la gestion fiscale complète d'une entreprise, nous apportons un accompagnement efficace dans la comptabilité en général (tenue de la comptabilité, établissement des salaires, de budgets, analyse financière et conseils, décomptes TVA, ...). Au bénéfice d'une bonne expérience dans le domaine immobilier, nous sommes actifs également dans la gérance d'immeubles et de PPE. Par ailleurs, notre société est à même de proposer un soutien à la formation administrative de votre personnel.

Appuyés par une équipe expérimentée parlant le français, l'italien, l'espagnol et l'anglais, nous offrons une large palette de services allant de la gestion des paiements, travaux administratifs divers à des problématiques de ressources humaines ou de création d'entreprise.

Nous nous réjouissons de vous rencontrer et de faire votre connaissance afin d'évoquer ensemble vos besoins.

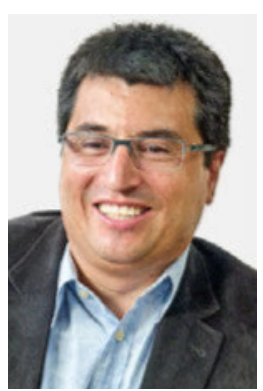

Mario Fedi

Spécialiste en finance et comptabilité avec brevet fédéral mario.fedi@fmhfiduciaire.ch

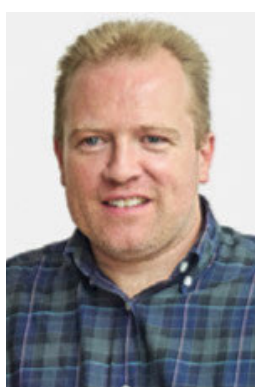

Yann Burgener

Spécialiste en finance et comptabilité avec brevet fédéral yann.burgener@fmhfiduciaire.ch
Finest Gestion Sàrl

FMH Services (Fiduciaire)

Faubourg Saint-Germain 2

2900 Porrentruy

Tél. 0329322400 - Fax 0329322402

finestgestion.ch
Finest Gestion Sàrl est une entreprise de conseil indépendante sur le plan juridique et économique recommandée par la société coopérative FMH Services.

\section{Problèmes de TVA?}

\section{T) FMHSERVICES}

«Les prestations médicales ne sont pas soumises à la TVA!» Si cette affirmation est correcte, elle ne l'est cependant pas dans tous les cas. C'est pourquoi il convient d'étudier la question en détail dans le cadre de cabinets doubles ou de groupe ou encore de la propharmacie. En tant que spécialistes, les partenaires de confiance FMH Services (Fiduciaire) se tiennent à votre entière disposition. N’hésitez pas à faire appel à eux.

\section{FIDUCIAIRE}

FMH Consulting Services AG

Tél. 0419250077 - Fax 0419250067

mail@fmhfiduciaire.ch - www.fmhfiduciaire.ch 